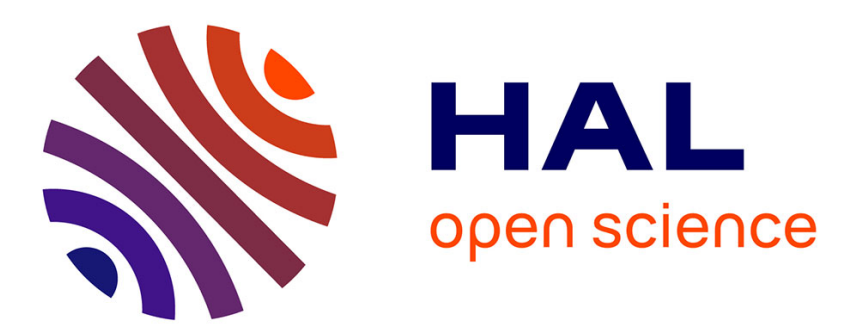

\title{
Mesure in situ de l'amplitude des ondes réfléchies ou rétrodiffusées par les sédiments marins. Application à la détermination in situ des modes granulométriques
}

\author{
J.P. Longuemard
}

\section{- To cite this version:}

J.P. Longuemard. Mesure in situ de l'amplitude des ondes réfléchies ou rétrodiffusées par les sédiments marins. Application à la détermination in situ des modes granulométriques. Revue de Physique Appliquée, 1978, 13 (9), pp.471-479. 10.1051/rphysap:01978001309047100 . jpa-00244477

HAL Id: jpa-00244477

https://hal.science/jpa-00244477

Submitted on 1 Jan 1978

HAL is a multi-disciplinary open access archive for the deposit and dissemination of scientific research documents, whether they are published or not. The documents may come from teaching and research institutions in France or abroad, or from public or private research centers.
L'archive ouverte pluridisciplinaire HAL, est destinée au dépôt et à la diffusion de documents scientifiques de niveau recherche, publiés ou non, émanant des établissements d'enseignement et de recherche français ou étrangers, des laboratoires publics ou privés. 


\title{
MESURE IN SITU DE L'AMPLITUDE DES ONDES RÉFLÉCHIES OU RÉTRODIFFUSÉES PAR LES SÉDIMENTS MARINS. APPLICATION A LA DÉTERMINATION IN SITU DES MODES GRANULOMÉTRIQUES
}

\author{
J. P. LONGUEMARD
}

\begin{abstract}
Centre de Recherches de sédimentologie marine de Perpignan, Centre Universitaire de Perpignan, avenue de Villeneuve, 66025 Perpignan, France
\end{abstract}

(Reçu le 24 novembre 1977, révisé le 26 avril 1978, accepté le 22 mai 1978)

\begin{abstract}
Résumé. - L'utilisation des ondes réfléchies sous incidence normale pour l'étude de l'impédance acoustique et des paramètres qui en découlent (densités, paramètres mécaniques) fait l'objet de nombreuses publications $[1,2,8,12]$. Le système émetteur récepteur des ondes est généralement placé à bord d'un navire océanographique ce qui impose des longueurs de trajets acoustiques importantes. Les fréquences utilisées sont souvent inférieures à $30 \mathrm{kHz}$ et les sonars génèrent les ondes à fréquence unique.

Le procédẻ présenté ci-dessous diffère des précédents car :

— il fonctionne à partir d'un bâti posé sur le fond marin. Cette disposition des sondes réduit au minimum le trajet des ondes acoustiques.

L'émission ultra-sonore se fait suivant un balayage en fréquence compris entre 20 et $100 \mathrm{kHz}$.

Des mesures effectuées in situ permettent de corréler la valeur des fréquences ultra-sonores pour lesquelles l'amplitude détectée passe par un maximum à la dimension des modes granulométriques des sédiments. Des sédiments sont prélevés par bennes sur les sites expérimentaux en vue d'analyses en laboratoire.
\end{abstract}

\footnotetext{
Abstract. - Reflection and bag scattering of ultra sound at normal incidence in marin sediments are usually used to determine their acoustical impedance and the characteristics of sound (density, percentage in water, shear strength). The waves are generated at the water surface from oceanographic ship. The acoustical way is long and the general frequencies are shorter than $30 \mathrm{kHz}$. The sonar products only one frequency.

This study describes an another method. The support of the piezoceramics down on the bottom of the sea and the acoustical way is short.

The acoustical waves are protected at the switching frequencies $(20$ and $100 \mathrm{kHz})$.

The data obtained in situ permit the correlations between the granulometric modes and the frequencies for which the amplitude of reflected waves is maximum. The granulometric modes are determined in vitro with sample of the sea floor extracted on the experimental stations.
}

1. Etude théorique. - Deux principes s'appliquent à l'étude de l'amplitude des ondes ultra-sonores renvoyées par les fonds sous-marins soumis à une onde incidente.

1.1 L'INTERFACE EAU-SÉDIMENT EST CONSIDÉRÉ COMME UN RÉFLECTEUR PARFAIT. - La valeur du coefficient de réflexion est alors définie par (Fig. 1) :

$$
R=\frac{Z_{2} \cos \theta_{\mathrm{i}}-Z_{1} \cos \theta_{\mathrm{r}}}{Z_{2} \cos \theta_{\mathrm{i}}+Z_{1} \cos \theta_{\mathrm{r}}}
$$

$Z_{2}$ et $Z_{1}$ sont les impédances acoustiques du sédi- ment (2) et de l'eau (1), $\theta_{\mathrm{i}}$ et $\theta_{\mathrm{r}}$ sont les angles incidents et réfractés.

Pour des vitesses de propagation des ondes $V_{2}$ et $V_{1}$ dans les milieux (2) et (1) $\theta_{\mathrm{i}}$ et $\theta_{\mathrm{r}}$ valent :

$$
\frac{V_{1}}{V_{2}}=\frac{\sin \theta_{\mathrm{i}}}{\sin \theta_{\mathrm{r}}}
$$

1.2 L'INTERFACE SE PRÉSENTE SOUS UNE FORME RUGUEUSE (FIG. 2). - Dans ce cas les grains de sable situés sur le plan de séparation rayonnent une onde diffuse. L'énergie diffusée par chaque source est faible devant l'énergie de l'onde incidente. 


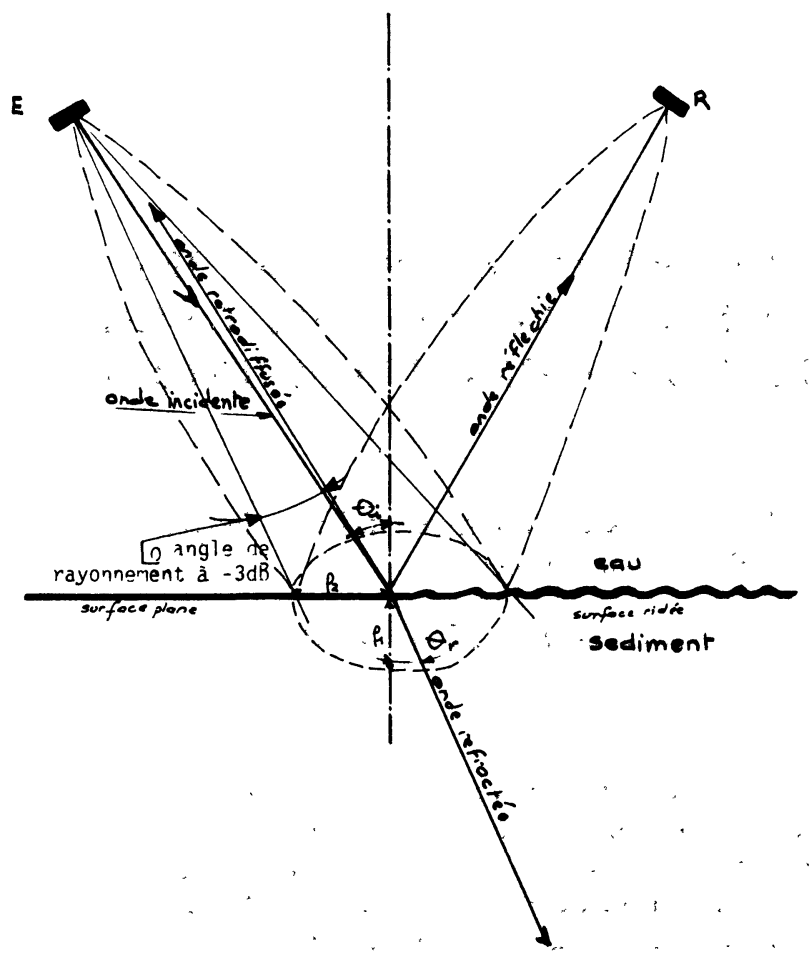

FIG. 1. - Comportement d'une onde incidente à l'interface eausédiment.

[Reflection refraction and scattering waves from the ocean bottom.]

Pour une onde incidente définie par :

$$
\psi_{\mathrm{i}}=\mathrm{e}^{j k r} \cos v
$$

qui agit sur une surface composée de $N$ grains (Fig. 2), le niveau de l'onde diffuse reçue par un capteur situé en $P$ devient [10] :

$$
\psi_{\mathrm{r}}=\sum_{n=1}^{N} \frac{f\left(v_{n}\right)}{R_{n}} \exp \left[j k_{0} \cdot r_{n}+k R_{n}\right]
$$

$f\left(v_{n}\right)$ est fonction de la distribution des grains de sable et de leurs dimensions.

Dans la pratique, il est impossible de connaître la fonction de distribution spatiale. Outre l'incertitude

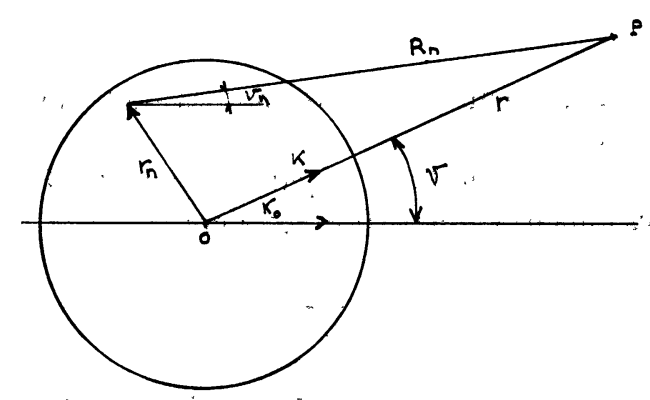

Fig. 2. - Influence d'un ensemble de générateurs d'ondes diffuses (angles position et nombre d'ondes).

[Angles, position and propagation vectors for scattering of plane wave by ensemble of scatters.] sur la distribution des réflecteurs il y a lieu de tenir compte :

- des pertes d'énergie sur les parcours acoustiques,

- des variations de la surface de sol sous-marin étudiée provoquées par les modifications des lobes de rayonnement des sondes émettrices-réceptrices (§2).

Les fonds marins se présentent souvent sous l'aspect d'une surface ondulèe et la rếflexion des ondes se fait sur une section limitée par l'intersection du lobe de rayonnement à $3 \mathrm{~dB}$ d'angle au sommet $2 \theta$ et le fond marin (Fig. 1).

La valeur de $\theta$ est définie par [6], l'équation (5) pour une source émettrice circulaire plane de diamètre $d$ supérieur à $\lambda / 2$ :

$$
\theta=\arcsin \frac{1,4 \lambda}{\pi d}
$$

La vitesse de propagation des ondes ultrasonores dans l'eau de mer est de l'ordre de $1460 \mathrm{~m} / \mathrm{s}$ et varie en fonction de la salinité et de la température, de ce fait, $\lambda=c / f$ varie légèrement.

Pour les essais effectués (cf. $\S 2$ ), les diamètres des faces parlantes sont de 6 et $7 \mathrm{~cm}$. Les valeurs respectives des angles $\theta$ en fonction de $f$ sont représentées par les fonctions ci-dessous :

$$
\begin{aligned}
& \theta_{1}=\arcsin \cdot \frac{15622}{f} \\
& \theta_{2}=\arcsin \cdot \frac{18270}{f}
\end{aligned}
$$

La figure 3 traduit les variations de $\theta$ en fonction de $f$. Compte tenu de la précision des essais en nature nous pouvons admettre, avec une erreur de l'ordre de $1,5 \%$, que :

$$
\begin{array}{ll}
\theta_{1}^{0}=10^{6} / f, & \theta_{1} \mathrm{rad}=1,74 \times 10^{4} / f \\
\theta_{2}^{0}=1,16 \times 10^{6} / f, & \theta_{2} \mathrm{rad}=2,02 \times 10^{4} / f .
\end{array}
$$

Pour un sédiment unimodal de distribution gaussienne, de mode $\bar{m}$ et d'écart type $\sigma$, le nómbre de grains de dimension $m$ compris sur une surface' $S$ est proportionnel à :

$$
N_{\text {grains }}=\frac{S}{\bar{m}^{2}} \cdot \exp -\frac{(m-\bar{m})^{2}}{2 \sigma^{2}} .
$$

Or la surface de forme elliptique, soumise à une onde incidente sphérique pour un émetteur donné, décroît avec la fréquence.

$S$ est fonction de la distance $r$ qui sépare la source émettrice du centre de la zone soumise aux ultrasons (Fig. 1).

Pratiquement, cf. calcul annexe, pour $f$ supérieur à $2 \times 10^{4} \mathrm{~Hz}, S$ vaut :

$$
S \# \frac{\pi r^{2}}{\sin \theta_{i}} \cdot \frac{3,3 \times 10^{8}}{f^{2}} .
$$




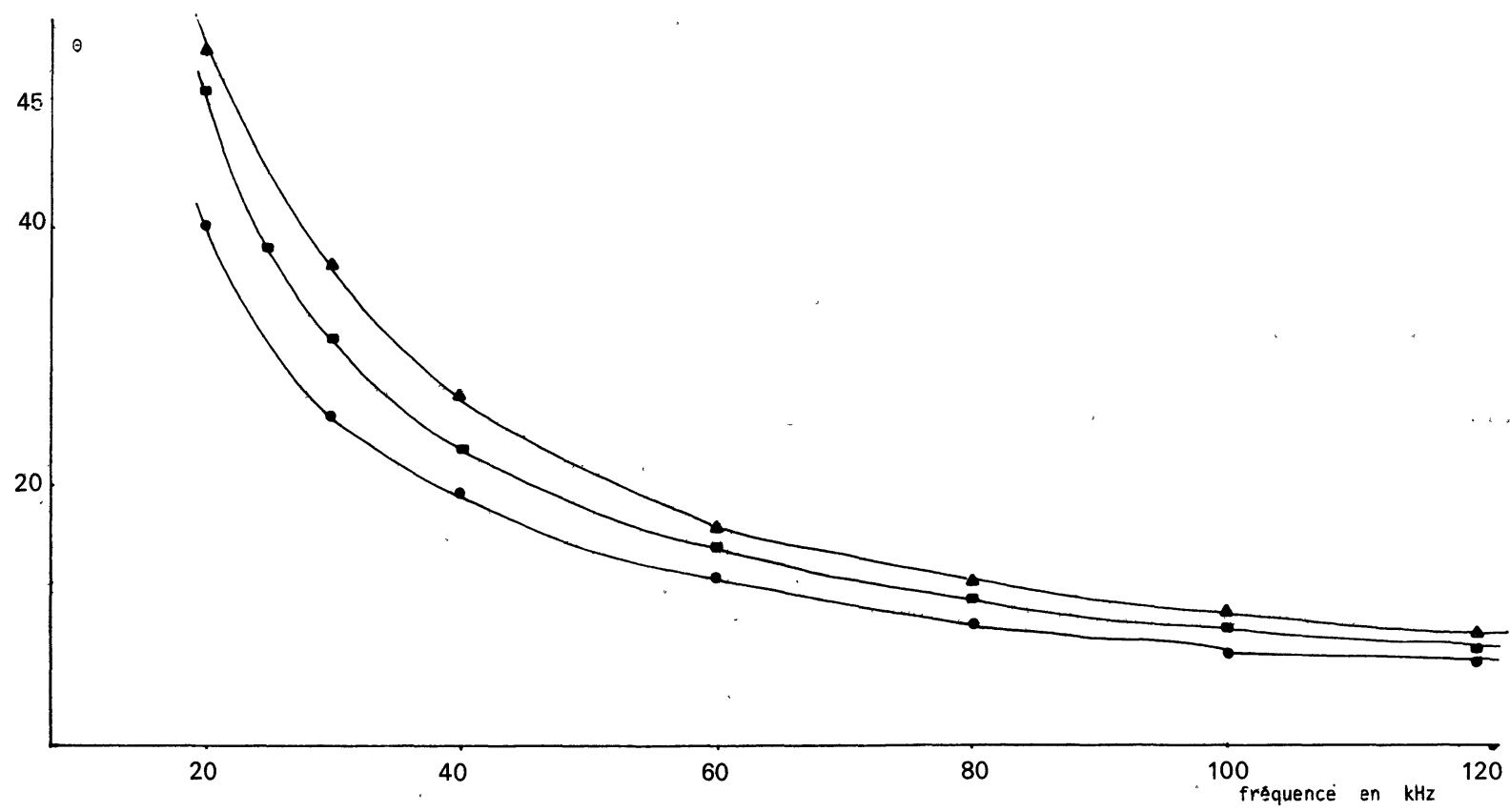

Fig. 3. - Variations du lobe de rayonnement en fonction de la fréquence.

[Variation of radiation lobe with frequency.]

$\theta=\operatorname{Arcsin} \frac{k d}{f} ; \quad \Delta d=7 \mathrm{~cm} ; \quad \boldsymbol{\quad} d=6 \mathrm{~cm} ; \quad \bullet d=5 \mathrm{~cm}$.

Finalement le nombre de grains de dimension $m$ influant sur $\psi_{\mathrm{r}}$ est défini par:

$N_{\text {grains }}=\frac{\pi r^{2}}{\sin \theta_{i}} \cdot \frac{3,3 \times 10^{8}}{f^{2}} \cdot \frac{1}{\bar{m}^{2}} \cdot \exp -\frac{(m-\bar{m})^{2}}{2 \sigma^{2}}$.

Notons par :

$$
g\left(\frac{2 \pi m f}{c}\right) N \equiv f(v)
$$

l'amplitude de l'onde diffúsée et écrivons $\psi_{\mathrm{r}}$ compte tenu de (9) et (10), pour un sédiment multimodal :

$$
\begin{aligned}
\psi_{\mathrm{r}}=\sum_{n} A_{n} & \cdot g\left(\frac{2 \pi m f}{c}\right) \cdot \frac{r^{2}}{f^{2}} \cdot \frac{1}{\bar{m}_{n}^{2}} \times \\
& \times \exp -\frac{\left(m-\bar{m}_{n}\right)^{2}}{2 \sigma_{n}^{2}} \exp \left(-\frac{\alpha f r}{2}\right) \cdot \frac{1}{r}
\end{aligned}
$$

où :

$A_{n}$ est un coefficient de proportionnalité,

$\sum$ doit en particulier tenir compte de la possibilité d'existence d'un sédiment multimodal, $n \in(1,2,3, \ldots)$, $\alpha$ est le coefficient d'atténuation des ondes.

Cette fonction montre que lamplitude reçue est étroitement liée à $f, m, \bar{m}$ et $r$.

Le but recherché est de proposer une relation liant $f$ et $\bar{m}$ lorsque $\psi_{\mathrm{r}}(\omega)$ présente des extréma.

La dérivée totale de $\psi_{\mathrm{r}}$ pour $n=1$,

$\mathrm{d} \psi_{\mathrm{r}}=\frac{\partial \psi_{\mathrm{r}}}{\partial r} \mathrm{~d} r+\frac{\partial \psi_{\mathrm{r}}}{\partial m} \mathrm{~d} m+\frac{\partial \psi_{\mathrm{r}}}{\partial \bar{m}} \mathrm{~d} \bar{m}+\frac{\partial \psi_{\mathrm{r}}}{\partial f} \mathrm{~d} f$, se réduit, au cas où seul $\bar{m}$ et $f$ sont des variables, à :

$$
\mathrm{d} \psi_{\mathrm{r}}=\frac{\partial \psi_{\mathrm{r}}}{\partial \bar{m}} \mathrm{~d} \bar{m}+\frac{\partial \psi_{\mathrm{r}}}{\partial f} \mathrm{~d} f
$$

Pour $m=\bar{m}$ et pour un sédiment unimodal, les dérivées partielles par rapport à $\bar{m}$ et à $f$ s'écrivent :

$$
\begin{aligned}
& \frac{\partial \psi_{\mathrm{r}}}{\partial \bar{m}}=A\left[\frac{2 \pi}{c} f \cdot g_{\bar{m}}^{\prime}\left(\frac{2 \pi \bar{m} f}{c}\right) \cdot \frac{r}{f^{2} \cdot \bar{m}^{2}}-\right. \\
& \left.-\frac{2}{\bar{m}^{3}} \frac{r}{f^{2}} g\left(\frac{2 \pi \bar{m} f}{c}\right)\right] \cdot \exp -\frac{\alpha r}{2} f \\
& \frac{\partial \psi_{\mathrm{r}}}{\partial f}=A\left[\frac{2 \pi \bar{m}}{c} g_{f}^{\prime}\left(\frac{2 \pi \bar{m}}{c} f\right) \cdot \frac{r}{f^{2} \bar{m}^{2}}-\right. \\
& \left.-\frac{2}{f^{3}} \frac{r}{\bar{m}^{2}} g\left(\frac{2 \pi \bar{m}}{c} f\right)-\frac{\alpha r^{2}}{2 f^{2} \bar{m}^{2}}\right] \cdot \exp -\frac{\alpha r}{2} f \text {. }
\end{aligned}
$$

Pour que $\psi_{\mathrm{r}}$ passe des extréma, il est nécessaire que $\partial \psi_{\mathrm{r}} / \partial \bar{m}$ et $\partial \psi_{\mathrm{r}} / \partial f$ soient nulles, ce qui a lieu si :

$$
\begin{aligned}
& \frac{g_{\bar{m}}^{\prime}\left(\frac{2 \pi \bar{m} f}{c}\right)}{g\left(\frac{2 \pi \bar{m}}{c} f\right)}=\frac{c}{\pi f \bar{m}} . \\
& \frac{g_{f}^{\prime}\left(\frac{2 \pi \bar{m} f}{c}\right)}{g\left(\frac{2 \pi \bar{m}}{c} f\right)}=\frac{c}{\pi f \bar{m}}+\frac{c \alpha r}{4 \pi} .
\end{aligned}
$$


Soit :

$$
\frac{g_{\bar{m}}^{\prime}\left(\frac{2 \pi \bar{m}}{c} f\right)}{g\left(\frac{2 \pi m f}{c}\right)}+\frac{g_{f}^{\prime}\left(\frac{2 \pi \bar{m}}{c} f\right)}{g\left(\frac{2 \pi m f}{c}\right)}=\frac{2 c}{\pi f \bar{m}}+\frac{c \alpha r}{4 \pi} .
$$

Dans l'hypothèse de la diffusion de Rayleigh, où la dimension $m$ d'un grain de sable est petite devant la longueur d'onde $\lambda$, l'énergie de l'onde diffusée est proportionnelle à $m^{6} f^{4}$, on prendra pour amplitude une fonction de la forme :

$$
g\left(\frac{2 \pi \bar{m} f}{c}\right)=\bar{m}^{3} f^{2} .
$$

Dans cette hypothèse la condition (18) s'écrit :

$$
\begin{aligned}
3 \log \bar{m}+2 \log f & =\frac{c \log \bar{m}}{\pi f}+ \\
& +\frac{c \log f}{\pi \bar{m}}+\frac{c \alpha r}{4 \pi} f+K(\bar{m}, f)
\end{aligned}
$$

ou encore :

$$
\begin{aligned}
\log \bar{m}\left(3-\frac{c}{\pi f}\right) & =\frac{c \alpha r}{4 \pi} f+ \\
& +\log f\left(-2+\frac{c}{\pi \bar{m}}\right)+K(\bar{m}, f) .
\end{aligned}
$$

$K(\bar{m}, f)$ étant une constante d'intégration, la relation (20) fait intervenir les paramètres $\alpha$ et $r$ qui dépendent des conditions d'essais en nature, les valeurs respectives de ces différents paramètres sont : $1460 \mathrm{~m} / \mathrm{s}, 2 \times 10^{6}$ nepers $/ \mathrm{m} / \mathrm{Hz}$ et $0,5 \mathrm{~m}$.

Elles permettent d'écrire (20) sous la forme :

$$
\begin{aligned}
\varphi(\bar{m})= & \frac{12 \pi}{1460 \alpha r} \log \bar{m}= \\
& =f+K^{\prime}(\bar{m}, f) \text { soit } 26 \log \bar{m}+a=f
\end{aligned}
$$

pour $f$ exprimé en $\mathrm{kHz}$ après avoir négligé les termes petits devant l'unité.

Pratiquement on peut considérer que $\psi_{\mathrm{r}}$ est maximum lorsque $f$ est lié à $\bar{m}$ par une relation approchée de la forme $a+b \log \bar{m}=f$. En effet, les calculs présentés ici ne tiennent pas compte de tous les paramètres salinité-turbidité-température qui influent sur la célérité et l'atténuation du son dans l'eau de mer donc sur la fonction $\varphi(\bar{m})$.

2. Principe de la mesure. - Les ondes diffusées par un sphéroïde sont omnidirectionnelles. Nous disposons de deux procédés de mesures pour étudier la fonction $f=\varphi(\bar{m})$ : mesures des ondes réfléchies ou mesures des ondes rétrodiffusées (Fig. 1). Le principe de fonctionnement du matériel est le même dans les deux cas : émettre des impulsions ultrasonores de fréquences variables, détecter les échos renvoyés par le fond, traiter et enregistrer le signal capté.

La figure 4 représente le schéma synoptique des circuits électroniques. Les pressions acoustiques et les

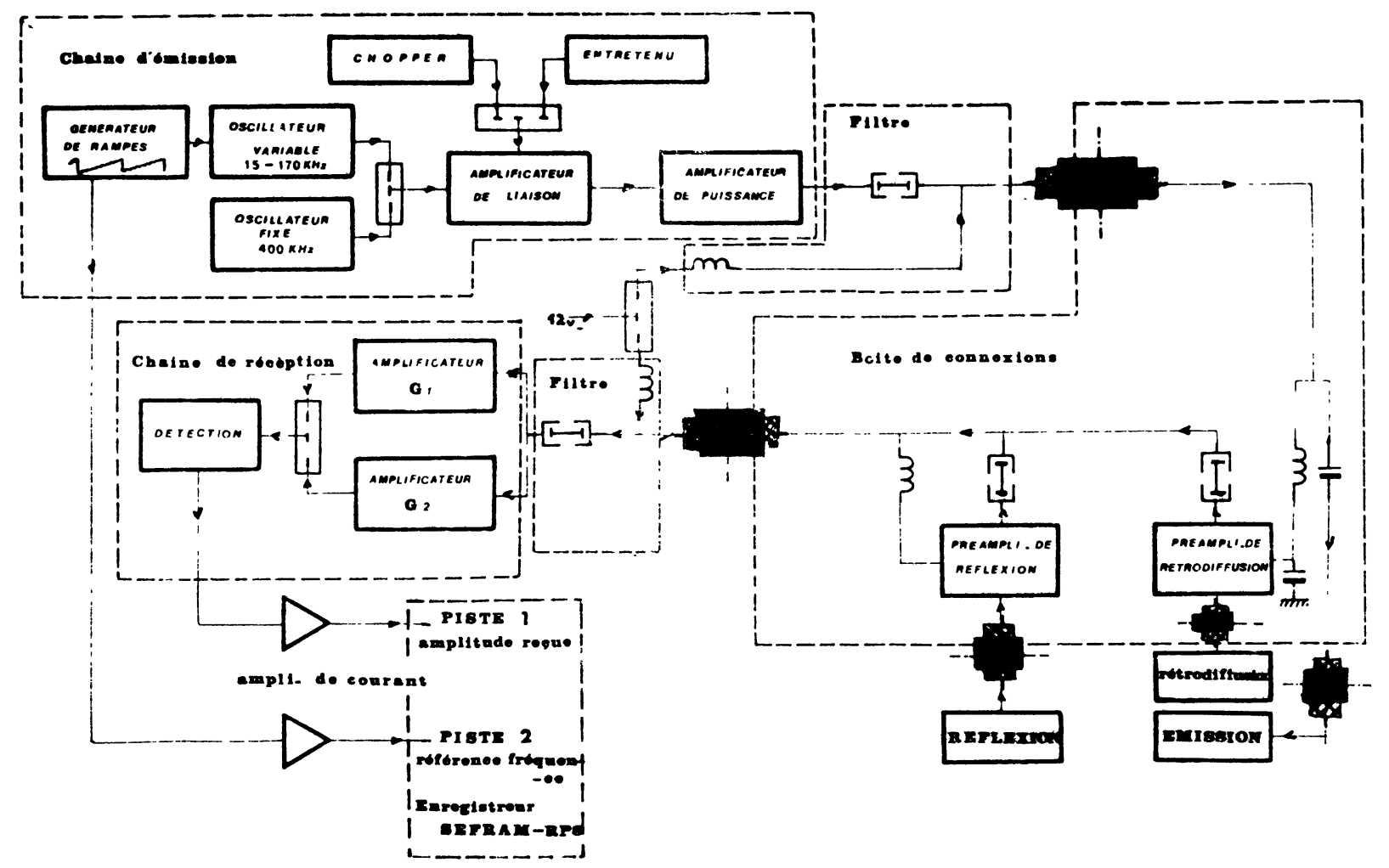

FIG. 4. - Schéma synoptique de la chaîne de mesure. 
tensions électriques appliquées aux transducteurs piézoélectriques déterminent un fonctionnement linéaire des sondes. Les tensions électriques mesurées sont donc proportionnelles aux pressions acoustiques $[4,5,9]$. La fonction de réponse des circuits émetteurs-récepteurs n'est pas linéaire; il est nécessaire au cours de l'exploitation des résultats de corriger les données acquises pour obtenir une variation de $V_{\mathrm{r}}=h(\omega)$ proportionnelle à $\psi_{\mathrm{r}}$, indépendante de la bande passante des sondes.

3. Analyse des résultats acquis. - Nos nombreuses expériences in situ sur fonds sableux ont confirmé la possibilité d'extraire les valeurs des modes granulométriques à partir des variations de la fonction $V_{\mathrm{r}}=h(\omega)$. La figure $5 a$ et $b$ illustre un exemple de l'influence de l'histogramme granulométrique sur $\psi_{\mathrm{r}}$.



Fig. 5a. - Exemple de variations de l'amplitude de $V_{\mathrm{r}}=f(\omega)$.

[Example of waves amplitude variation $V_{\mathrm{r}}=f(\omega)$.]

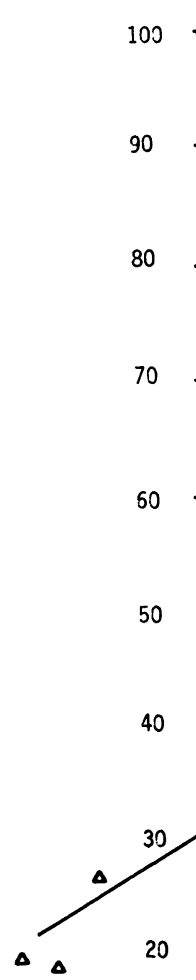

$\mathrm{F}$ en $\mathrm{KHz}$

Réflexion $\theta=40^{\circ}$

$\mathrm{S}_{\mathrm{e}}=28 \mathrm{~cm}^{2}$

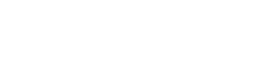

2

$\Delta$

Les sédiments multimodaux fournissent une valeur extrême de $\psi_{\mathrm{r}}$ par mode. Plusieurs séries d'essais conduits à partir du même matériel électronique mais
$\Delta \Delta$

$\Delta$

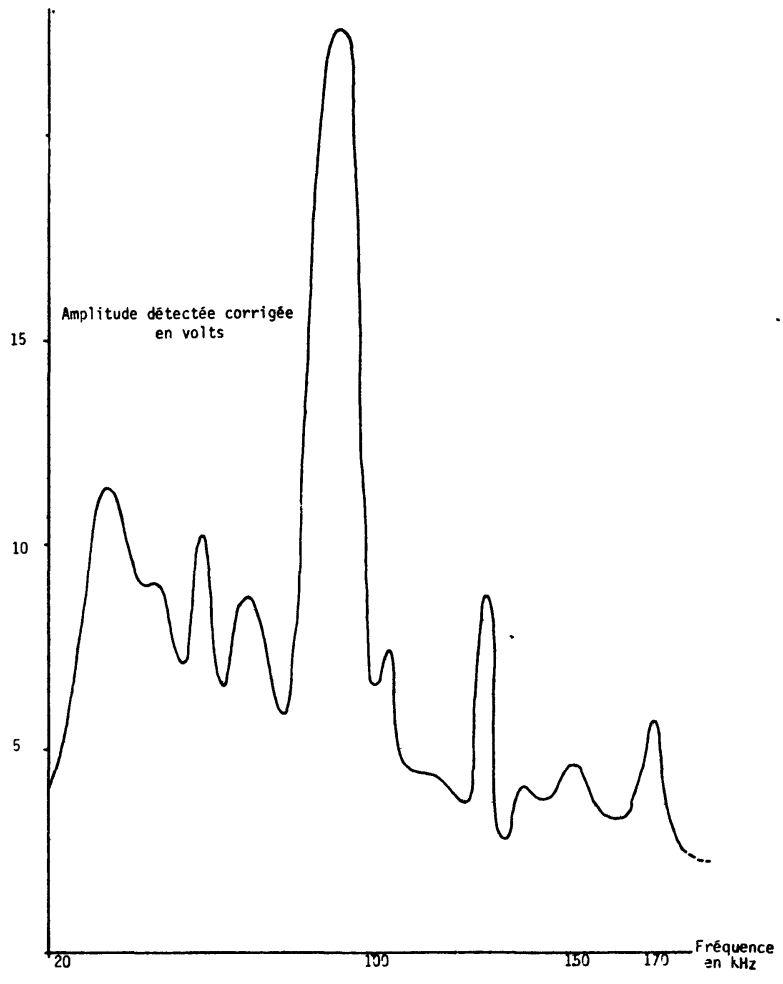

Fig. 5b. - Histogramme granulométrique.

[Differential granulometric curve.]

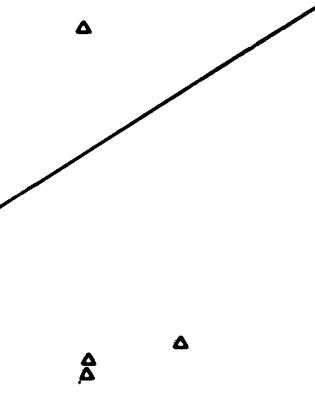

$\hat{\mathbf{\Delta}}$

Fig. $6 a$ 
avec des angles incidents et des surfaces de sondes émettrices fournissent des données compatibles (Figs. $6 a$ à $e$ ) avec la relation (20) soit :

$$
\begin{array}{lrl}
f= & 8+23,5 & \log \bar{m} \\
f= & 25 & \log \bar{m} \\
f= & 26 & \log \bar{m} \\
f= & 10+23 & \log \bar{m} .
\end{array}
$$

\section{$f$ est exprimé en $\mathrm{kHz}, \bar{m}$ est exprimé en $\mu$.}

Chacune de ces formules expérimentales établies par une analyse statistique (régression logarithmique) correspond :

- pour la relation (21), à un angle incident de $40^{\circ}$ et une surface émissive de $28 \mathrm{~cm}^{2}$,

- pour la relation (22), à un angle incident de $20^{\circ}$ et une surface émissive de $28 \mathrm{~cm}^{2}$,

- pour la relation (23), à un angle incident de $40^{\circ}$ et une surface émissive de $38 \mathrm{~cm}^{2}$,

- pour la relation (24), à une onde rétrodiffusée suivant une rasance de l'ordre de $30^{\circ}$ et une surface émissive de $38 \mathrm{~cm}^{2}$.

Globalement pour des angles incidents de 20 et $40^{\circ}$, la relation (20) devient (Fig. 6e) :

$$
f=25 \log \bar{m} \text {. }
$$

La différence entre les valeurs des constantes $a$ et $b$ s'explique par l'importance de la surface $S_{0}$ qui est fonction de l'incidence (ou rasance) et de l'angle du lobe de rayonnement principal. Ces données montrent que pour cette série de mesure, plus $S_{0}$ est faible, plus la valeur numérique de $b$ est élevée.

L'application des variations de l'amplitude des ondes réfléchies et rétrodiffusées à la détermination des modes granulométriques est confirmée par les équations (23) et (24). La comparaison entre les modes granulométriques calculée à partir de (23) et (24) et les modes mesurés en laboratoire par tamisage ou par sédimentométrie sur des échantillons prélevés par bennes sur les sites expérimentaux fournie un facteur de corrélation supérieur à 0,9 pour les deux cas.

L'écart constaté entre les valeurs des termés $a$ et $b$ des formules (21 à 24) est provoqué par une légère différence entre les surfaces des sédiments soumises aux ultrasons.

Le calcul de $\bar{m}$ à partir de la fonction $f=a+b \log \bar{m}$ est définie par :

$$
\bar{m}=\exp 2,3 \frac{(f-a)}{b} .
$$

Deux facteurs influent sur la précision de ce calcul : - la connaissance de $f$ pour laquelle $\psi_{\mathrm{r}}$ est maximum,

- la valeur de $b$ qui est inversement proportionnel à $\alpha$.

Les variations de $\alpha$ ont un rôle prédominant, en effet, pour une variation de la température de l'eau de 5 à $22^{\circ} 5, \alpha$ donc $b$ varie dans un rapport de l'ordre de 2 [6]. La fréquence $f$ est fixée à $\pm 2,5 \mathrm{kHz}$ quelle que

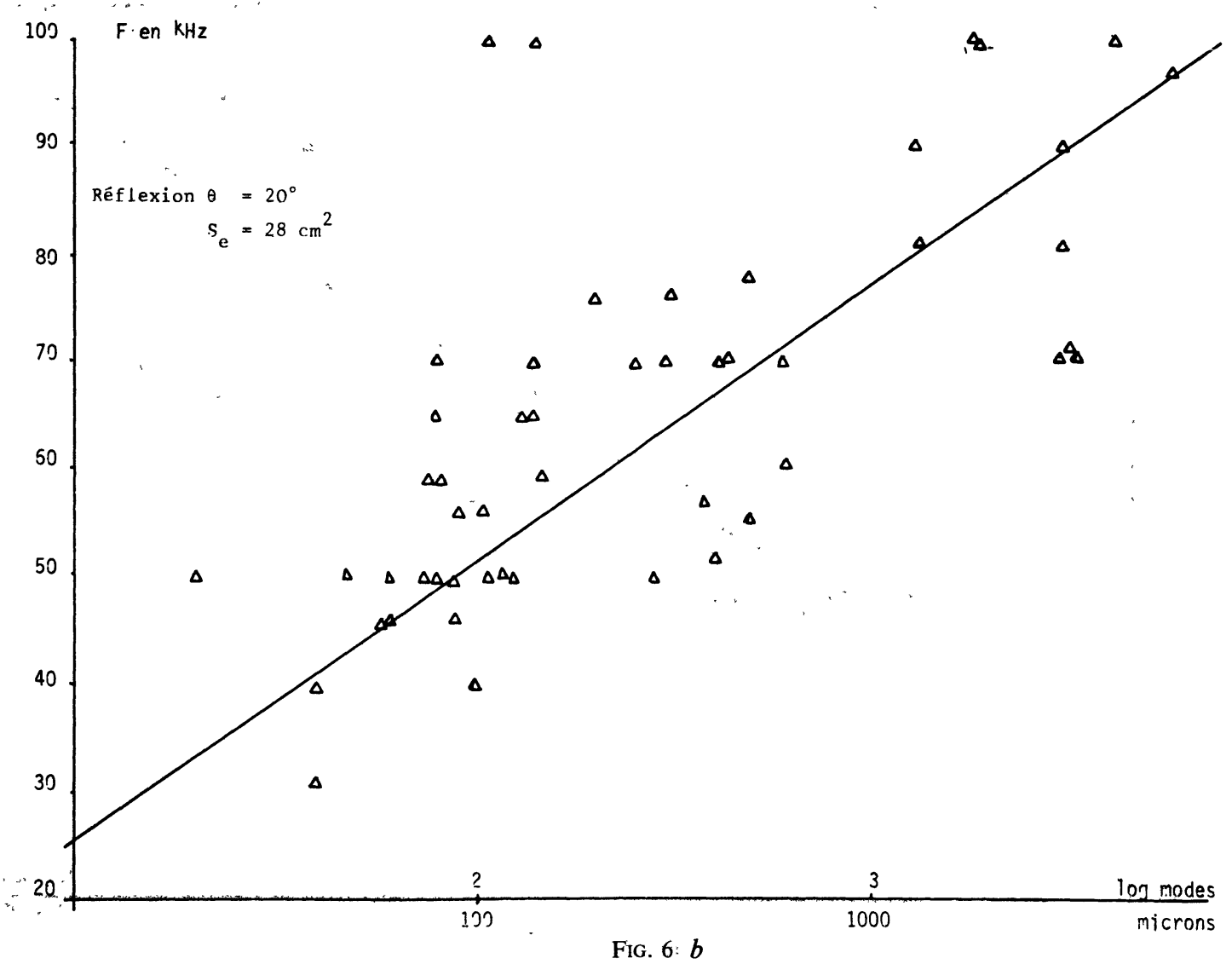

FIG. 6: $b$ 


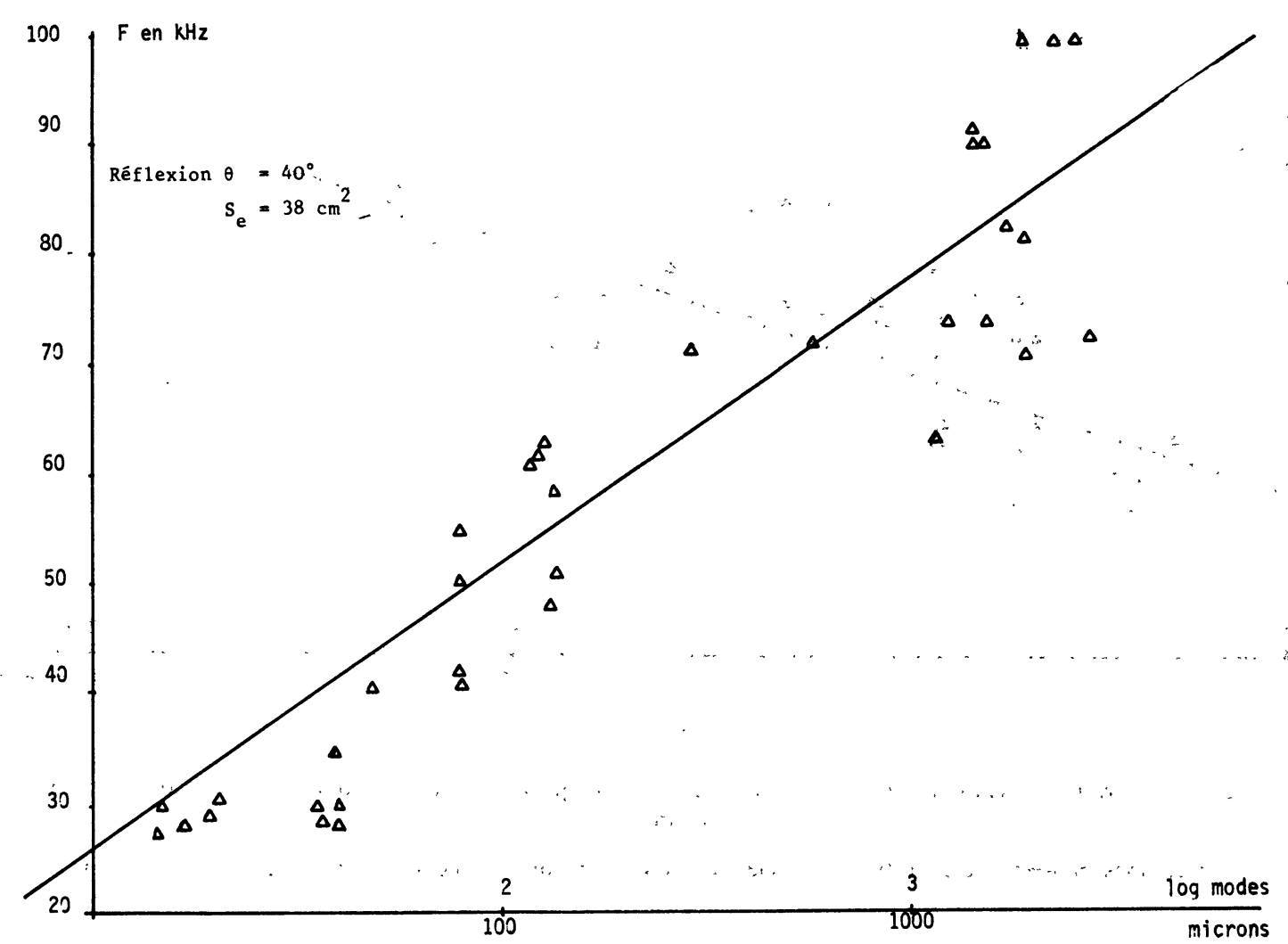

Fig. $6 c$

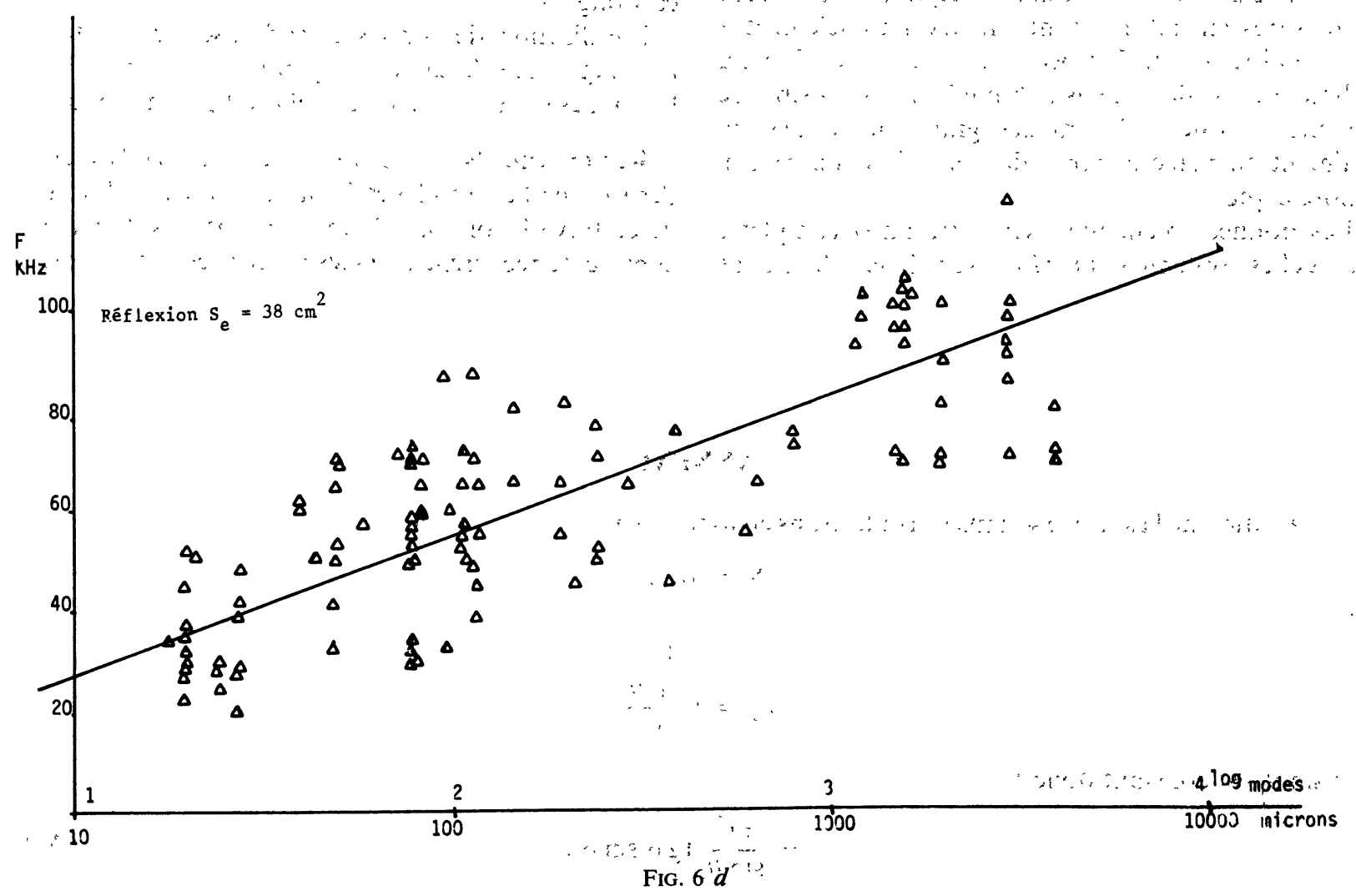






FIG. $6 e$

Figs. $6 a, b, c, d, e .-$ Corrélations entre les fréquences acoustiques pour lesquelles $V_{\mathrm{r}}$ est maximum et la dimension des modes granulométriques.

[Correlations between the acoustic frequencies which $V_{\mathrm{r}}$ is maximum and the granulometrics grains size.]

soit la valeur de $f$. Dans ces conditions, $\log \bar{m}$ est calculé à $35 \%$. Le matériel permet néanmoins de connaître le nombre de modes présents sur le site étudié ainsi que leur répartition dimensionnelle.

4. Conclusion. - Les résultats acquis par ce procédé de mesures en nature montrent que parallèlement à la connaissance de l'impédance acoustique $\left(Z_{2}\right)$ du sédiment donc de sa densité humide, il est possible de calculer la valeur des modes granulométriques du sédiment en place à partir des variations du niveau sonore capté.

Les données recueillies, sans être aussi complètes que celles obtenues in vitro sur échantillons, ne nécessitent aucune opération ultérieure. L'utilisation de ce matériel à partir d'un vecteur circulant sur le fond ou à son voisinage permettrait de réaliser des cartes sédimentologiques sous-marines en tracé continu. Ces mesures facilitent l'interpolation entre chaque station expérimentale ponctuelle (cas des dragages ou carottages).

L'utilisation des ondes rétrodiffusées présente un avantage dans le cas où l'opérateur souhaite placer les sondes sur un vecteur se déplaçant près du fond.

Remerciements. - L'auteur remercie la Direction des Recherches, Etudes et Techniques qui est à l'origine de ce travail, son aide financière a permis de réaliser la présente recherche dans les meilleures conditions.

\section{ANNEXE}

Variation de la surface soumise aux ultrasons en fonction de $f$.

où :

$$
S=\pi \rho_{1} \cdot \rho_{2}
$$

$$
\begin{aligned}
& \rho_{1}=r \operatorname{tg} \theta \\
& \rho_{2}=r \frac{\sin \theta}{\sin \theta_{i}}
\end{aligned}
$$

or : $\sin \theta_{\mathrm{i}}$ est constant donc :

$$
S=\frac{\pi r^{2}}{\sin \theta_{\mathrm{i}}} \operatorname{tg} \theta \sin \theta
$$


Le développement limité de $\operatorname{tg} \theta$ et de $\sin \theta$, permet d'écrire :

$$
\begin{aligned}
& S=\frac{\pi}{\sin \theta_{i}} \cdot r^{2} \cdot\left[\frac{\theta-\frac{\theta^{3}}{3 !}+\frac{\theta^{5}}{5 !}+(-1)^{n} \frac{\theta^{2 n+1}}{(2 n+1) !}}{1-\frac{\theta^{2}}{2 !}+\frac{\theta^{4}}{4 !}+(-1)^{n} \frac{\theta^{2 n}}{2 n !}}\right] \cdot\left[\theta-\frac{\theta^{3}}{3 !}+\frac{\theta^{5}}{5 !}+(-1)^{n} \frac{\theta^{2 n+1}}{(2 n+1) !}\right] \\
& S=\frac{\pi}{\sin \theta_{\mathrm{i}}} r^{2} \cdot \dot{\theta}\left[\frac{1-\frac{\theta^{2}}{3 !}+(-1)^{n} \frac{\theta^{2 n}}{(2 n+1) !}}{1-\frac{\theta^{2}}{2 !}+(-1)^{n} \frac{\theta^{2 n}}{2 n !}}\right] \cdot\left[\theta-\frac{\theta^{3}}{3 !}+(-1)^{n} \frac{\theta^{2 n+1}}{(2 n+1) !}\right] .
\end{aligned}
$$

Compte tenu des valeurs de $\theta$ et les fréquences ultrasonores utilisées $S_{0}$ varie comme :

$$
S=\frac{1,1 \pi}{\sin \theta_{i}} r^{2} \cdot\left[\theta^{2}-\frac{\theta^{4}}{3 !}+(-1)^{n} \frac{\theta^{(2 n+2)}}{(2 n+1) !}\right] .
$$

En remplaçant $\theta$ par sa valeur en fonction de $f$, la surface $S$ pour une sonde de $6 \mathrm{~cm}$ de diamètre a pour expression :

$$
S \# \frac{1,1 \pi r^{2}}{\sin \theta_{\mathrm{i}}}\left[\left(\frac{1,74 \times 10^{4}}{f}\right)^{2}-\frac{1}{6}\left(\frac{1,74 \times 10^{4}}{f}\right)^{4}+\frac{1}{120}\left(\frac{1,74 \times 10^{4}}{f}\right)^{6}+\cdots\right] .
$$

\section{Bỉbliographie}

[1] Bezdeck, H. F., J. Geophys. Res. 78 (1973) 3390-3394.

[2] CLAY, C. S., Scattering and reflection of acoustica waves at the bottom and surface of the ocean. University Research Center Madison Final Report no 74-1, 50 p., 1974.

[3] Dragonette, L. R., Vogth, R. H., Flaw, L. et Neubauer, W. G., II transient Analysis. J. Acoust. Soc. Am. 5 (1974) 1130-1137.

[4] Gagnepain, J. J., Ann. Fr. de chrono et micro 10 année (1972) 10-21.

[5] Gallego, J. A., Inarez, Y. et Rodriguez, C., Analysis mediante circuite equivalente de un emison ultra sonoro con placa vibrante. Electron. Fis. Apli. 16 (1973) 605-616.

[6] Gueysse, Sabate, P., Acoustique sous-marine (Dunod) 1964, p. 236.

[7] GREN, R. F., IEEE Trans. Audio and Electroacoust. AU 21 (1973) $407-412$
[8] Knott, S. T., Hartley Huskins, E., Reflection energetics and acoustics impedance of the seabed from normal incidence. Seismic reflection woods Hole Oceanographic Institution. Massachusetts 02543, 1976.

[9] LoNंguemard, J. P., J. UOF VI, bull. no 1 (1974).

[10] MoRSE, M. et FESBACH, H., Methods of theoretical physics (Mac Graw Hill Bool Company Part I et II) 1953.

[11] ShoR, G. G., RaItT, R. W., MAC GowaN, D., Seismic refraction studies in the southern California Bordelan. Scripps Institution of Oceanography San Diego, California. SIO Réf. 76-13, 1976.

[12] Weltorn, P. J., Frey, H. G. et Moore, P., Experimental measurement of the scattering of acoustic waves by rough surface. J. Acoust. Soc. Am. 52 (1972) 1553-1563.

[13] W̄illiams, A. O., Acoustic reflection from a structured sea Bottom. J. Acoust. Soc. Am. 59 (1976) 62-68. 